\title{
Erythrocytes are Produced in Thick Membrane-Like Structures in Adult Mouse Blood
}

\section{Kong $\mathbf{W}^{*}$, Zhu XP, Han XJ and Wang $\mathbf{H}$}

Beijing Khasar Medical Technology Co. Beijing, Shangdi Donglu, Beijing, China

"Corresponding author: Kong W, Beijing Khasar Medical Technology Co. Beijing, Shangdi Donglu, Beijing, China, Tel: 086-010-82781147; E-mail: wuyikong@yahoo.com

Received date: July 20, 2018; Accepted date: July 31, 2018; Published date: August 03, 2018

Copyright: (C) 2018 Kong W, et al. This is an open-access article distributed under the terms of the Creative Commons Attribution License, which permits unrestricted use, distribution, and reproduction in any medium, provided the original author and source are credited.

\begin{abstract}
Erythrocyte-restricted progenitors are believed to have self-renewal capabilities. However, a recent study did not detect daughter pairs of erythrocyte progenitors, which questions the self-renewal ability of erythrocyte-restricted progenitors. In adult mouse blood, we identified a group of dense-eosin-stained thick membrane-like cellular structures that were from 30 to $400 \mu \mathrm{m}$ in diameter and called them "erythrocyte sacs". Erythropoiesis was initiated inside these sacs and terminated when the sac membrane broke to release hemoglobin+ and CD34+ functional erythrocytes. Numerous erythrocytes were produced, enucleated and released from one sac, which explains why the paired daughter cells were not detectable, and why the number of erythrocytes is about 1000 times higher than the sum of other blood cells. After erythrocytes were released, the thick sac membrane became fiber-like and degraded. Our data provide evidence that erythropoiesis occurs inside these erythrocyte sacs. These sacs do not renew and they produce only erythrocytes and no other types of blood cells.
\end{abstract}

Keywords: Erythrocyte; Hemoglobin; Terminally differentiated; Erythropoiesis; Enucleation

\section{Introduction}

Erythropoiesis is the process by which progenitors of erythrocytes are produced and terminally differentiate. The classical consensus is that in postnatal life, hierarchical differentiation occurs in the bone marrow, from hematopoietic stem cells (HSCs) that are defined by a self-renewal ability to stably reconstitute life-long blood components [1-5]. The hierarchical differentiation of HSCs first results in common myeloid progenitors and common lymphoid progenitors. Common myeloid progenitors then differentiate into granulocyte monocyte progenitors and megakaryocyte erythroid progenitor, the latter further segregating into erythrocyte and megakaryocyte progenitors [6,7]. This hierarchical differentiation process was defined on the basis of an immuno-phenotypic definition of HSCs collected by cell sorting, mostly determined by the activation of lineage-specific transcription factors such as C/EBPa, PU.1, and GATA-1 and expression of surface proteins such as CD150, CD34, and CD41 [8-10].

Recently, the classical consensus of the hierarchical differentiation of HSCs has been challenged. Using more vigorous and thorough experimental methods, studies described that except for platelet-biased stem cells that stay at the apex of the hierarchy $[11,12]$, in adulthood, the differentiation of HSCs to other blood progenitors features a twotier hierarchy; that is, HSCs directly differentiate to unipotent progenitors that are the predominant components in bone marrow $[13,14]$. These reports claim that except for the megakaryocyte lineage, the HSCs in the hematopoietic compartment contain a group of mixed unipotent progenitors [15]. The new concept has also been supported by somatic cell studies describing that multipotent progenitors in the embryonic period become unipotent progenitors in adulthood $[16,17]$. In general, these reports challenged the classical consensus and led to a new consensus that either HSCs or somatic stem cells in adults are lineage-restricted or unipotent progenitors.

Although reports have provided evidence for the hematopoietic compartment containing an aggregation of unipotent progenitors, most still consider that these unipotent progenitors may have selfrenewal capabilities to reconstitute life-long blood components [15]. However, in a series of experiments, Yamamoto et al. [8] failed to detect the paired daughter cells of erythroid progenitors despite detecting the paired daughter cells of other blood cell progenitors, which questioned whether erythroid progenitors have self-renewal ability.

In the current study, we investigated adult mouse blood and found numerous mature erythrocytes released in a synchronized pattern from novel cellular structures we call "erythrocyte sacs". Our findings provide novel evidence for erythropoiesis in adulthood.

\section{Methodology}

\section{Animals and materials}

Male Balb/C and c57BL6 mice were purchased from Vital River Laboratories (Beijing). Mice received food and water ad libitum. The animal protocol titled "Examination of stem cells in mouse blood" was approved by Beijing Khasar Medical Technology Animal Regulatory Office. All animal procedures followed the guidelines of the Animal Regulatory Office relevant to national and international guidelines. Anti-CD34 antibody was from Santa Cruz Biotechnology (Santa Cruz, CA, USA). Anti-hemoglobin antibody was from Abcam (Shanghai). Alexa Fluor goat anti-rabbit and donkey anti-mouse IgG antibodies were from Invitrogen (Carlsbad, CA, USA). 


\section{Mouse blood collection}

Blood was collected each time from 5 of 8 - to 10 -week-old Balb/C or 8- to 10 -week-old c57BL/6 mice via cardiac puncture under sterile conditions after CO2 euthanasia. Each syringe was prefilled with $100 \mu \mathrm{l}$ heparin $(0.5 \%)$ to prevent coagulation. Blood was pooled $(\sim 5 \mathrm{ml})$ and transferred to a $50-\mathrm{ml}$ centrifuge tube, immediately diluted with $1 \mathrm{x}$ phosphate buffered saline (PBS) at a ratio of 1:5 (blood:PBS), then centrifuged at $200 \mathrm{xg}$ for $5 \mathrm{~min}$. After removing the supernatant, the cellular portion was diluted by PBS in a 1:1 ratio, then $15 \%$ paraformaldehyde was slowly added to a final concentration of $4 \%$ paraformaldehyde. Blood cells were fixed at $4^{\circ} \mathrm{C}$ for overnight.

The next day, cells were centrifuged at 200xg for $5 \mathrm{~min}$, then washed 2 times with PBS and centrifuged at $100 \mathrm{xg}$ for $5 \mathrm{~min}$ and twice at $50 \mathrm{xg}$ for $5 \mathrm{~min}$ to remove free erythrocytes. Visible red clots were removed during washing. After a final centrifugation and removing the supernatant, the cellular portion was fixed again with $4 \%$ paraformaldehyde at a ratio of 1:5 (original blood volume:fixative volume) and dropped ( $200 \mu \mathrm{m} / \mathrm{drop}$ from $\sim 5 \mathrm{~cm}$ above) onto gelatinecoated histology slides.

This method yielded 2 to 5 erythrocyte sacs on one slide although most were not in good condition for taking images. At least 20 slides from each strain of mice were stained and examined.

\section{Fluorescence staining}

Slides were blocked for $1 \mathrm{hr}$, incubated with anti-CD34 and antihemoglobin antibodies in blocking buffer overnight at $4^{\circ} \mathrm{C}$, then washed and incubated with a fluorescent-conjugated secondary antibody for $1 \mathrm{hr}$. After a washing, cells were counterstained with DAPI and photographed by conventional fluorescence microscopy (Leica, Wetzlar, Germany) and photographed with use of a digital camera (Leica, DFC500). Control tissue was treated with non-specific antiserum from the same species of primary antibody.

\section{Hematoxylin and eosin (H\&E) staining}

Cellular portions from each mouse strain preparation were dropped on at least 10 slides. Slides were dried overnight, washed 3 times with PBS, and underwent H\&E staining. Stained slides were visualized under a standard fluorescence microscopy (Leica) and photographed with use of a digital camera (Leica, DFC500).

\section{Results}

\section{Identifying dense-eosin-stained "erythrocyte sacs"}

On H\&E-stained histology slides, many dense-eosin-stained "erythrocyte sacs" were identified by their distinct red color and rare morphology that has never been described for other blood components. These sacs were large, ranging from $30 \mu \mathrm{m}$ (Figure 1A and $1 \mathrm{~B}$ ) to $>200 \mu \mathrm{m}$ in diameter (Figure $1 \mathrm{C}$ and $1 \mathrm{D}$ ).

Some were thick membrane-like and almost $400 \mu \mathrm{m}$ in diameter (Figure 1E). Each had a thick grey-colored membrane that could be easily identified at the periphery. The grey-colored continuous membrane indicated that these structures were not cell colonies nor from aggregation of erythrocytes.

However, some thick membrane-like sacs (Figure 1E-1H), which stained weakly for eosin, may be a more primitive sac than those in Figure 1, because they contained numerous reticular materials that were the markers of reticulocytes or the immature form of erythrocytes.

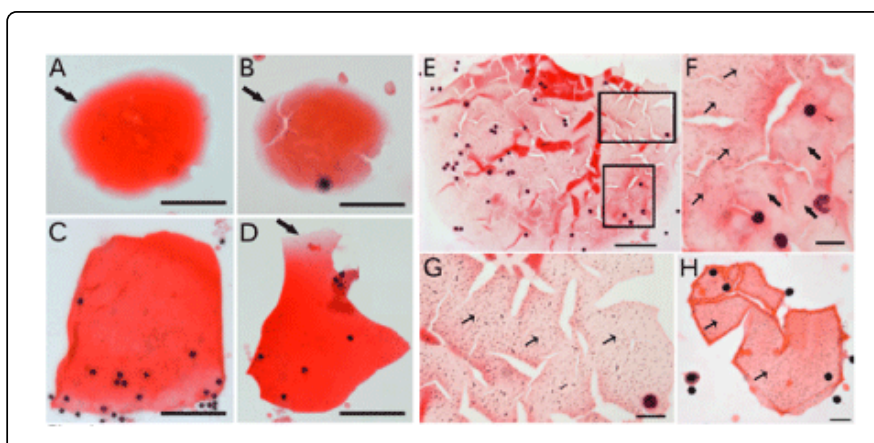

Figure 1: Identifying "erythrocyte sacs". Dense-eosin-stained cellular structures were observed on histology. The images were taken with $100 \mathrm{x}(\mathrm{A}, \mathrm{B})$ or $40 \mathrm{x}$ lenses $(\mathrm{C}, \mathrm{D})$. Structures in A or B were about 30 to $40 \mu \mathrm{m}$ in diameter and in $\mathrm{C}$ or $\mathrm{D}$ were about 150 to $200 \mu \mathrm{m}$ in diameter. Arrows point to the thick grey membrane at the periphery of each sac. A weak-eosin-stained membrane structure $\sim 400 \mu \mathrm{m}$ in diameter (E) had evenly distributed reticular structures except for a small area (lower rectangle in E). Amplified images showed areas with reticular structures (wide-head arrows in F), without reticular structures (thick arrows in F) or all reticular structures (arrows in $\mathrm{G})$. (H) Evenly distributed reticular structures in a membrane fragment (arrows). Bars in $A-B=20 \mu \mathrm{m} ; \mathrm{C}-\mathrm{D}=50 \mu \mathrm{m} ; \mathrm{E}=50 \mu \mathrm{m}$; F$\mathrm{H}=10 \mu \mathrm{m}$.

\section{Each erythrocyte sac contained tightly packed contents}

In our preliminary studies, except for eosin or Giemsa staining (Supplementary Figure S1), most immuno-stains were negative for specific proteins on the membrane of these erythrocyte sacs, possibly because the thick membrane is not a typical cellular membrane. As well, the thick membrane of some sacs became transparent and separated on one side, which resulted in a clam-shaped sac (Figure 2A).

This shape may have been caused by the method of dropping samples on slides during slide preparation or by expansion of the contents when they become differentiated. The tightly packed contents in the erythrocyte sacs were exposed, but higher magnification revealed that most of the contents did not have the features of standard erythrocytes, except for a few located at the periphery (Figure $2 \mathrm{~B}$ and 2C).

Higher magnification also revealed that the separated membrane was almost jelly-like and transparent and was still attached to the content portion of the sac (Figure 2D). Hemoglobin expressed only on a small portion of the contents at periphery (Figure 2E) of one sac (Figure $2 \mathrm{~F}$ ), which suggests that the contents in this sac were immature erythrocytes.

Some contents showed almost no expression of hemoglobin (Supplementary Figure S2), which suggests that this sac was more primitive than the one in Figure 2A. 


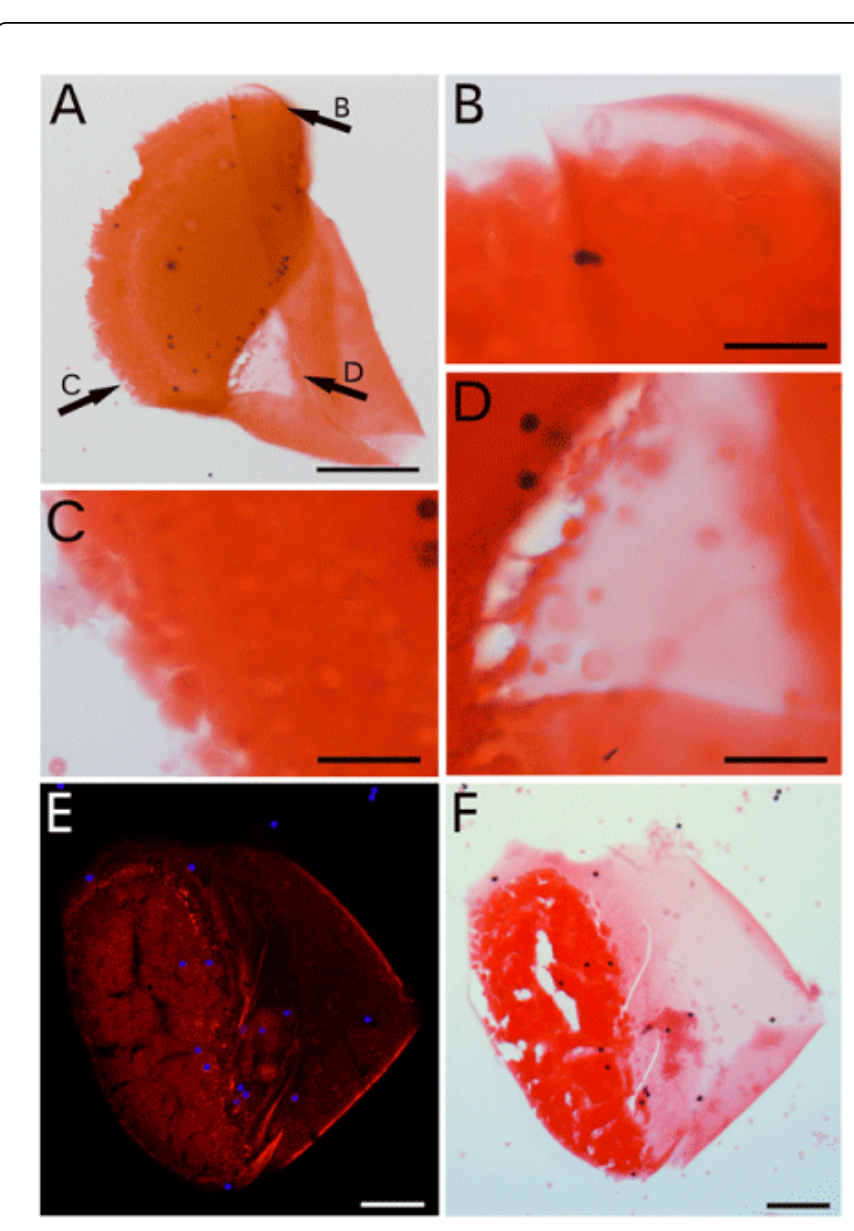

Figure 2: The outer membrane of the erythrocyte sac can separate from the cell contents. A large dense-eosin-stained sac had a clamlike shape and was half-covered by a jelly-like membrane (A). Amplified images (arrows in A) showed that the jelly-like membrane covered part of this sac (B); the materials located at periphery of the tightly packed contents exhibited the morphology of erythrocytes (C); and the semi-transparent membrane was attached to the side containing tightly packed contents (D). Hemoglobin expressed on the contents at the periphery in one sac (E). H\&E stain (F) revealed that half of the sac in (E) was a thick membrane (F). Bars in $\mathrm{A}=100 \mu \mathrm{m}$; $\mathrm{B}-\mathrm{D}=20 \mu \mathrm{m}$; in $\mathrm{E}-\mathrm{F}=50 \mu \mathrm{m}$.

\section{Erythropoiesis occurs inside the erythrocyte sacs}

Although we did not develop any useful techniques to isolate or purify these erythrocyte sacs from blood to further examine them systemically in vitro, we considered that a "living body" could function as an incubator in that the differentiation status of each erythrocyte sac would not be the same. Because of the unique morphology of these erythrocyte sacs, the "living incubator" could be used to study the development of these erythrocyte sacs. Among many morphologically different sacs, here we provided images of 3 erythrocyte sacs that had similar staining and sizes (Figure 3 ). The eosin-stained density varied slightly on one sac (Figure $3 \mathrm{~A}$ ) in that the amplified image revealed several nuclei, each surrounded by a small circle (arrow in Figure 3A) located in the denser-stained areas. These nuclei may be halfway trapped inside the membrane or undergoing enucleation. Another sac (Figure 3B) had cracked lines with many "naked" nuclei located immediately adjacent, which suggests that these nuclei had been released or enucleated out of the sac. The third sac (Figure 3C) showed numerous erythrocytes with the typical disk-like morphology released from the broken membrane at one side of the sac. The amplified image (arrow in Figure 3C) revealed that these 2 nuclei did not have the shadowed circles of those in Figure 3A. The differences supported our suggestion that the nuclei in Figure 3A were halfway trapped inside the membrane. Our data provide evidence that erythropoiesis started from tightly packed materials to mature erythrocytes inside these erythrocyte sacs. This observation also provides evidence that numerous erythrocytes are produced in a synchronized pattern perhaps not via mitotic division. The mature erythrocytes only live for one "life", which explains why no blood diseases are caused by overgrowth of erythrocytes.

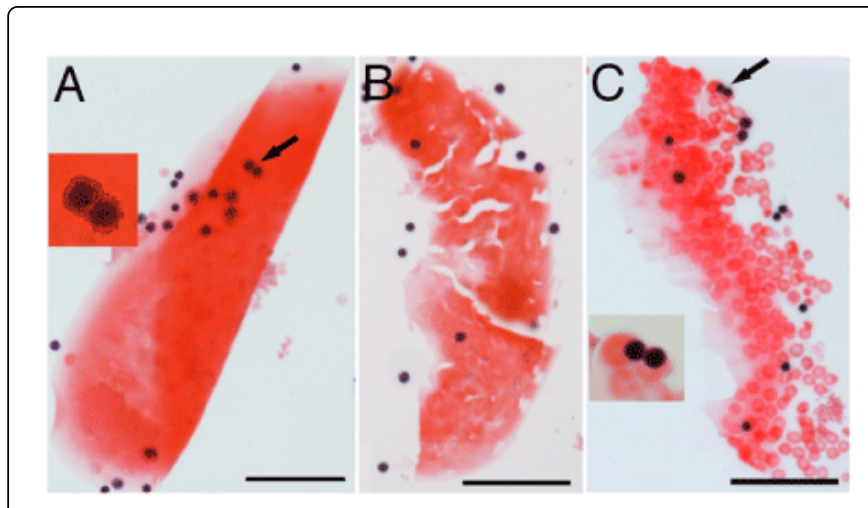

Figure 3: Erythropoiesis occurs inside the erythrocyte sacs. Three sacs with similar sizes and stains are presented. Image A shows a sac with several adjacent nuclei. Except for the "naked" nuclei, each nucleus is surrounded by a small ring-like shadow (arrows and amplified image in A). With a consistent membrane, the sac in (B) has cracked lines. The thick membrane in the third sac (C) was broken, and numerous erythrocytes were observed. A few nucleated erythrocytes (arrows and amplified image in $\mathrm{C}$ ) were identified. Bars in $\mathrm{A}-\mathrm{C}=50 \mu \mathrm{m}$.

\section{Mature erythrocytes were released from each sac}

We further tested the specific protein of erythrocytes, hemoglobin, in the sac contents that showed a standard disk-like erythrocyte shape. Hemoglobin and CD34 both expressed on the disk-like erythrocytes but in a differential pattern (Figure 4A). Almost all erythrocytes expressed hemoglobin, but only those located at the periphery expressed CD34. The large thick membrane became transparent jellylike and showed no specific staining. We also observed disk-shaped erythrocyte that were packed in large group expressed both hemoglobin and CD34 (Figure 4B), which provides evidence that the maturate erythrocytes expressed CD34, albeit transiently.

In addition, we observed numerous erythrocytes that attached to the fiber-like materials or membranes in the background where no free erythrocytes were observed (Figure 5). The thick jelly-like membranes may have further become fiber-like materials after releasing the maturated erythrocytes. We also believe that these fiber-like membranes can be further degraded in circulation, which further suggests that these erythrocyte sacs live for only one "life". 


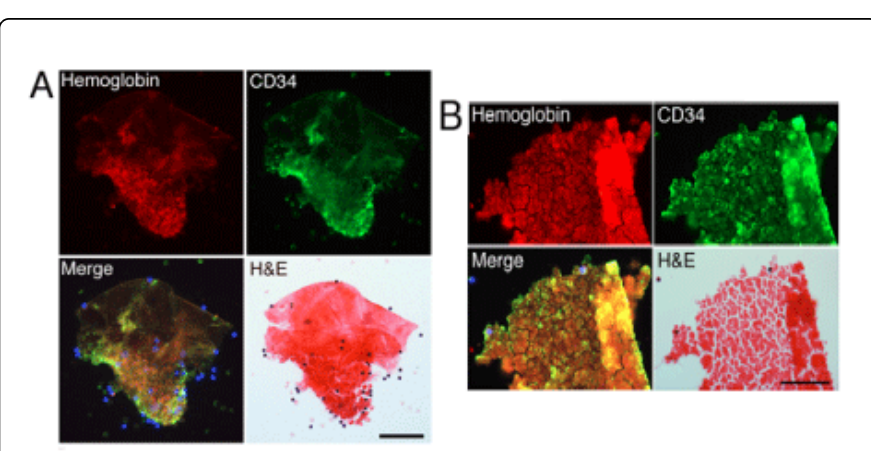

Figure 4: Hemoglobin+ and CD34+ erythrocytes were produced in erythrocyte sacs. The expression of hemoglobin and CD34 on erythrocytes in a sac examined by immunofluorescent staining. (A) Almost all erythrocytes stained positive for hemoglobin, and only these at the periphery stained positive for CD34. (B) Disk-like erythrocytes that packed together expressed both hemoglobin and CD34. The same slides were stained with H\&E after fluorescent images were taken. Bars $=50 \mu \mathrm{m}$.

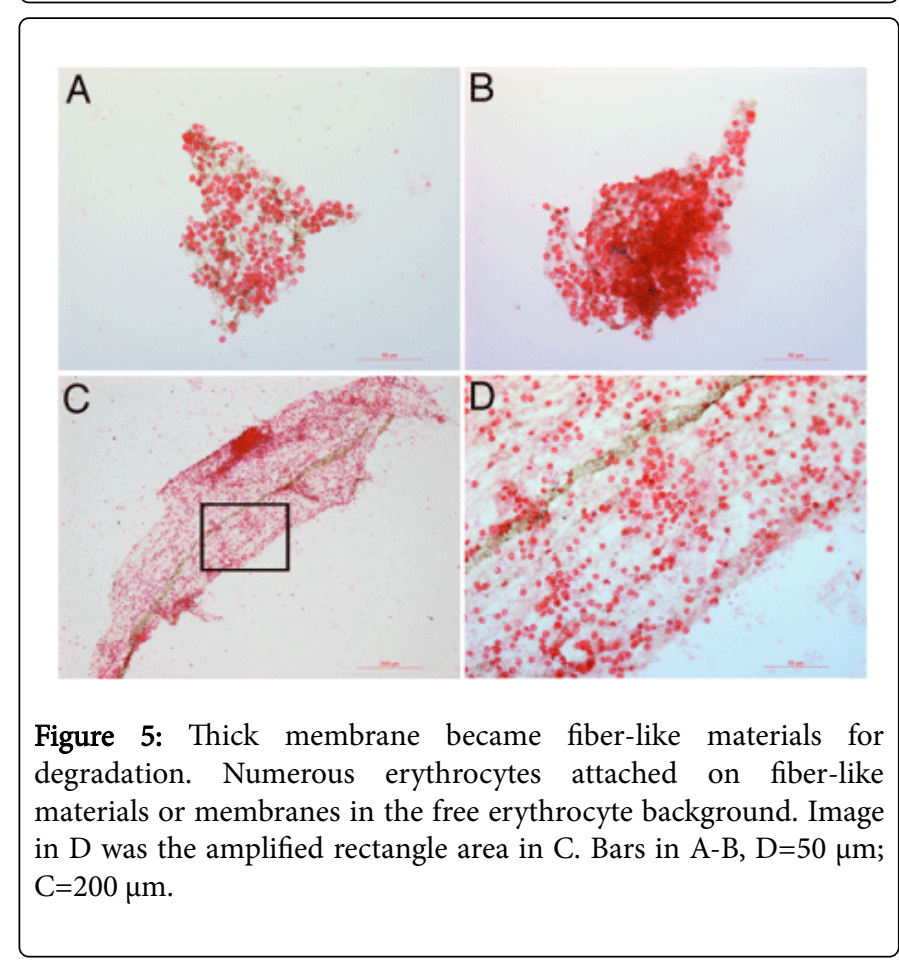

\section{Discussion and Conclusion}

Here we report the identification of the new type of cellular structures, erythrocyte sacs, for erythropoiesis in adult mouse blood. Our data reveal that hundreds or thousands of erythrocytes can be produced in a synchronized pattern inside one sac. This observation explains why the number of erythrocytes is about 1000 times higher than the sum of the other type of blood cells. Although relevant antibodies for the surface markers have been extensively used in cell biology for cell sorting and cell identification and have extremely sped up data acquisition, we used classical and time-consuming methods for this study. We believe that the larger membrane sizes could be left out of the sorting scale. Also, the thick coating membranes that do not express most well-known surface markers of the erythrocyte sacs may explain their not being identified by the regular immuno-phenotypic cell-sorting method.

We found that erythrocyte sacs produced only erythrocytes, which indicates that they are lineage-biased before erythropoiesis initiation. According to the hematopoietic consensus, some cells or proerythroblasts may be the prior forms of the erythrocyte sacs. Although we still have not identified the prior forms of the erythrocyte sacs or pro-erythroblasts that are believed to perform erythropoiesis, we questioned how the pro-erythroblasts could transform into the large structures with a thick coating that is not the standard cellular membrane. We doubt that the prior forms of the erythrocyte sacs belong to any standard cell types.

Both the erythrocyte sacs and the erythrocytes do not have selfrenewal capability, which explains why erythrocytes do not overgrow or cause cancer as do leucocytes [15]. Identifying the rich erythrocyte production of each erythrocyte sac also explains why the hematopoiesis is erythrocyte-biased. In conclusion, our current studies provide evidence for a unique process of erythropoiesis and also support the consensus that the HSC compartment contains a mixture of unipotent progenitors. However, we doubt whether the erythrocyte progenitors have standard cellular structures.

\section{Acknowledgment}

This work was supported by a grant from the Department of Technology, Inner Mongolia government, China. The authors thank Laura Smales (BioMedEditing) for English language editing.

\section{References}

1. Seita J, Weissman IL (2010) Hematopoietic stem cell: self-renewal versus differentiation. Wiley Interdiscip Rev Syst Biol Med 2: 640-653.

2. England SJ (2011) Immature erythroblasts with extensive ex vivo selfrenewal capacity emerge from the early mammalian fetus. Blood 117 : 2708-2717.

3. Shizuru JA, Negrin RS, Weissman IL (2005) Hematopoietic stem and progenitor cells: clinical and preclinical regeneration of the hematolymphoid system. Annu Rev Med 56: 509-538.

4. Wagers AJ, Christensen JL, Weissman IL (2002) Cell fate determination from stem cells. Gene Ther 9: 606-612.

5. Orkin SH, Zon LI (2008) Hematopoiesis: an evolving paradigm for stem cell biology. Cell 132: 631-644.

6. Nakajima H (2011) Role of transcription factors in differentiation and reprogramming of hematopoietic cells. Keio J Med 60: 47-55.

7. Heffner GC (2011) Novel hematopoietic progenitor populations revealed by direct assessment of GATA1 protein expression and cMPL signaling events. Stem Cells 29: 1774-1782.

8. Yamamoto R (2013) Clonal analysis unveils self-renewing lineagerestricted progenitors generated directly from hematopoietic stem cells. Cell 154: 1112-1126.

9. Nishikii H (2015) Unipotent megakaryopoietic pathway bridging hematopoietic stem cells and mature megakaryocytes. Stem Cells 33: 2196-2207.

10. Byrska-Bishop M (2015) Pluripotent stem cells reveal erythroid-specific activities of the GATA1 N-terminus. J Clin Invest 125: 993-1005.

11. Sanjuan-Pla A (2013) Platelet-biased stem cells reside at the apex of the haematopoietic stem-cell hierarchy. Nature 502: 232-236.

12. Carrelha J (2018) Hierarchically related lineage-restricted fates of multipotent haematopoietic stem cells. Nature 554: 106-111. 
Citation: Kong W, Zhu XP, Han XJ, Wang H (2018) Erythrocytes are Produced in Thick Membrane-Like Structures in Adult Mouse Blood. J Cytol Histol 9: 513. doi:10.4172/2157-7099.1000513

Page 5 of 5

13. Notta F (2016) Distinct routes of lineage development reshape the human blood hierarchy across ontogeny. Science 351: aab2116.

14. Grinenko $\mathrm{T}$ (2018) Hematopoietic stem cells can differentiate into restricted myeloid progenitors before cell division in mice. Nat Commun 9: 1898.

15. Mercier FE, Scadden DT (2015) Not all created equal: lineage hard-wiring in the production of blood. Cell 163: 1568-1570.
16. Wuidart A (2018) Early lineage segregation of multipotent embryonic mammary gland progenitors. Nat Cell Biol 20: 666-676.

17. Lilja AM (2018) Clonal analysis of Notch1-expressing cells reveals the existence of unipotent stem cells that retain long-term plasticity in the embryonic mammary gland. Nat Cell Biol 20: 677-687. 